\title{
De votos a escaños: un análisis \\ de los efectos del sistema electoral ecuatoriano
}

\section{From Votes to Seats: An analysis of the effects of the Ecuadorian electoral system}

\author{
Felipe Cisneros Palacios
}

Recepción: 28 de junio de 2013

Aceptación: 12 de agosto de 2013

\section{Resumen}

El artículo propone un análisis del sistema electoral ecuatoriano con base a tres efectos que este produce: la forma cómo los electores a través del voto expresan su preferencia por una organización política o un candidato; cómo estos votos se transforman en cargos públicos; y cómo estos resultados denotan la aplicación de los principios de paridad en la Asamblea Nacional del Ecuador. Los datos revelan que la mayoría de electores prefieren votar por los candidatos dentro de una sola lista, pero a la misma vez, los resultados dejan ver la complejidad del sistema, y la dificultad, bajo las reglas actuales de que mujeres ganen escańos en igual proporción que sus pares hombres.

Palabras clave: sistema electoral, circunscripción plurinominal, voto personalizado múltiple, paridad, escańos.

\begin{abstract}
This article puts forth an analysis of the Ecuadorian electoral system based on three effects produced by it: the way in which voters express their preference for a political organization or candidate, how votes become public seats, and how these results indicate the application of the principles of gender parity in the National Assembly of Ecuador. Statistics show that the majority of voters prefer to vote for candidates in the same political party. Data also shows the complexity of the system and the difficulty, with the current system of rules, for women to win as many seats as their male counterparts.
\end{abstract}

Keywords: electoral system, multi-member district, panachage, parity, seats. 
Los sistemas electorales desempeñan indudablemente un papel fundamental sobre el proceso politico. Tienen repercusiones sobre el comportamiento electoral y, de este modo, sobre la votación. Dieter Nohlen

\section{Introducción}

$\mathrm{E}$ I pasado 17 de febrero de 2013 se realizaron en el Ecuador elecciones generales para la designación de presidente y vicepresidente, asambleístas y parlamentarios andinos. Este proceso se caracterizó, entre otras cosas, por ser el primero en ser organizado por el Consejo Nacional Electoral bajo las disposiciones constitucionales vigentes (Constitución de la República, 2008) y bajo la normativa establecida en la Ley Orgánica Electoral y de Organizaciones Politicas de la República del Ecuador, Código de la Democracia (2009), ya que, para las elecciones generales de 2009, se aplicó la normativa establecida en el Capítulo Segundo del Régimen de Transición de la Constitución de la República, así como en la Ley Orgánica de Elecciones (2000) y demás leyes conexas vigentes al año 2009. Como resultado, en el 2013, los ecuatorianos eligieron un presidente y un vicepresidente, ciento treinta y siete asambleístas unicamerales entre representantes nacionales, provinciales y del exterior, y cinco parlamentarios andinos, quienes ejercerán funciones hasta el año 2017.

Los resultados de 2013, como todos los resultados electorales, dependen fundamentalmente de los efectos que producen los sistemas electorales, que dicho de paso son sistemas con particularidades muy propias a la realidad política de cada país. En el caso del Ecuador, por ejemplo, hay diseños específicos del sistema que solo se aplican en este país en comparación al resto de países del continente; tal es el caso del voto personalizado múltiple, o dicho de otra manera, la forma de votación que otorga la posibilidad al elector de indicar su preferencia por los o las candidatas de una sola lista o de varias listas hasta completar el número permitido para cada uno de los cargos a elegir. 
Sin embargo, los efectos del sistema electoral no solo influyen sobre los resultados electorales, sino también sobre aquellos principios constitucionales que afirman el derecho a la participación. Un caso específico es el requisito que exige la normativa electoral vigente a las organizaciones políticas ecuatorianas al momento de conformar sus listas pluripersonales; tanto partidos como movimientos políticos deben aplicar una distribución paritaria y alternada entre mujeres y hombres para todos los procesos de representación política; es decir, todas las listas que se presentan a elecciones en el Ecuador deben cumplir con una cuota de participación femenina del cincuenta por ciento y estar ordenadas de forma alterna por candidata mujer, candidato hombre, candidata mujer o viceversa ${ }^{1}$.

En este sentido, considerando al sistema electoral ecuatoriano como unidad de análisis y su afectación a los resultados electorales, sobre la base de los principios de paridad y alternabilidad, se observa una serie de posibilidades que tiene el elector ecuatoriano para definir sus preferencias electorales en el momento de ejercer su derecho al voto. Estas son: 1) el elector puede votar por todos los candidatos de una sola lista sin afectar la paridad y la alternancia de la lista; 2) el elector puede votar por un número menor al número permitido dentro de una sola lista afectando la paridad y la alternancia de la lista de acuerdo a sus preferencias (el elector bien puede votar solo por candidatos hombres o solo candidatas mujeres, o por hombres y mujeres pero sin completar el total de dignidades a elegir), en este caso el elector sigue votando por una línea partidista pero muestra sus preferencias por determinados candidatos dentro de la misma lista; 3 ) el elector puede votar entre listas hasta completar el número permitido para las dignidades a elegir afectando, de esta manera, la paridad y la alternancia de las candidaturas de acuerdo a sus preferencias (aquí también el elector puede votar solo por candidatos hombres o por candidatas mujeres, o por hombres

1 En Latinoamérica, las legislaciones de Ecuador, Bolivia y Costa Rica son las únicas que exigen la conformación paritaria y alterna en las listas pluripersonales, aunque el efecto real en la composición de la legislatura está por debajo del $40 \%$. Solo Argentina, con una cuota femenina del $30 \%$, cuenta con una legislatura integrada por el $42 \%$ de legisladoras mujeres (IFE, 2011). 
y mujeres), en este caso el elector no vota por una línea partidista ya que distribuye sus preferencias por más de una organización política; y 4) el elector puede votar entre listas sin completar el número permitido para las dignidades a elegir afectando, de igual forma, la paridad y la alternancia de las candidaturas de acuerdo a sus preferencias, este voto representa una preferencia hacia más de una línea partidista.

Estas posibilidades de voto invitan a indagar el comportamiento del elector en concordancia con las normas establecidas en el sistema electoral ecuatoriano y a analizar los efectos que el mismo sistema produce sobre las preferencias del elector, los resultados electorales y la posibilidad real de que mujeres y hombres accedan a espacios de representación en forma paritaria. Es por esto que es necesario profundizar sobre los componentes constitutivos que integran este sistema electoral: el tipo y tamańo de las circunscripciones electorales, las formas de candidaturas y votación, y las fórmulas de conversión de votos en escaños; más aún cuando los resultados - y por tanto los derechos de participación- dependen de la interacción e interrelación de estos componentes.

El presente análisis investiga el sistema electoral ecuatoriano, en particular los tres componentes que lo conforman a partir de la votación del electorado de la provincia de Pichincha. El análisis toma como referencia los resultados electorales y los datos relativos a una investigación realizada por la Delegación Provincial Electoral de esta provincia. Se evidencia la forma cómo el electorado expresa sus preferencias a través del voto, así mismo, cómo influye el tipo y el tamaño de las circunscripciones y las fórmulas de asignación de escaños en los resultados finales y, finalmente, cómo los efectos que provienen del sistema electoral repercuten sobre la posibilidad real de representación paritaria en la Asamblea Nacional.

El artículo se divide en cuatro instancias: parte con una descripción de la metodología seleccionada para el análisis; continúa con aproximaciones teóricas y normativas de los componentes que estructuran a los sistemas electorales, en especial al sistema electoral ecuatoriano vigente; posteriormente se analiza la expresión del voto con base en la mues- 
tra de la provincia de Pichincha, así como los efectos que produce el sistema sobre los resultados electorales legislativos 2013, considerando principalmente el acceso de mujeres y hombres a la Asamblea Nacional; y finaliza con conclusiones y recomendaciones orientadas a fortalecer la toma de decisiones de las autoridades electorales del Ecuador en esta materia.

\section{Metodología}

En esta investigación se ha elaborado un análisis cuantitativo a partir de los datos obtenidos en el marco de un proyecto liderado y financiado por la Delegación Provincial Electoral de Pichincha ${ }^{2}$ (2013), el cual tuvo como punto de partida el diseño de un cuestionario de ciento noventa y cuatro preguntas, conducido por el Departamento de Capacitación de la institución, denominado Formulario para Análisis de Paquete Electoral. El cuestionario se diseñó con la finalidad de obtener una aproximación estadística que permita, por un lado, mejorar la gestión operativa de la Delegación Provincial Electoral y, por otro lado, analizar las trascendencias del sistema electoral desde la expresión del voto de esta provincia. Se han utilizado datos oficiales relativos a la inscripción de candidaturas del proceso electoral 2013, resultados electorales nacionales y aquellos concernientes a la provincia de Pichincha, además de los datos del cuestionario contenidos a partir de la pregunta 127 en adelante ${ }^{3}$.

Es importante resaltar que el proyecto seleccionó una muestra aleatoria de quinientos paquetes electorales, lo que corresponde al $7 \%$ de

2 Se felicita la iniciativa de los funcionarios María Elena Rizzo y Diego Tello quienes, junto al personal técnico de la Delegación Provincial Electoral de Pichincha, idearon y ejecutaron el proyecto del cual se elabora el análisis. Así mismo, se agradece la colaboración del equipo de veinte personas quienes recolectaron la información de los paquetes electorales y tabularon la información sobre la base de los datos que reposan en la delegación.

3 Agradezco la colaboración de Esteban Rosero, funcionario del Instituto de la Democracia, por su aporte con los datos estadísticos que se presentan en las tablas y el gráfico de este artículo. 
un total de siete mil ciento once juntas receptoras del voto (JRV): la muestra consideró una representación equitativa de paquetes electorales por sexo, circunscripción electoral, parroquia y zona electoral, asegurando de esta manera información proporcional en cuanto a electoras mujeres, hombres y territorio ${ }^{4}$. La siguiente tabla resume el total de papeletas seleccionadas para la muestra.

\section{Tabla 1.}

Total de papeletas seleccionadas para la muestra

\begin{tabular}{c|c|c|c|c|c}
\hline JRV & Total & $\%$ & $\begin{array}{c}\text { Asambleístas } \\
\text { nacionales }\end{array}$ & $\begin{array}{c}\text { Asambleístas } \\
\text { provinciales }\end{array}$ & $\begin{array}{c}\text { Parlamentarios } \\
\text { andinos }\end{array}$ \\
\hline Mujeres & 254 & 50,8 & 68296 & 68627 & 68653 \\
\hline Hombres & 246 & 49,2 & 64816 & 64849 & 64658 \\
\hline TOTAL & 500 & 100 & 133112 & 133476 & 133311 \\
\hline
\end{tabular}

Fuente: Delegación Provincial Electoral de Pichincha (2013)

Elaboración: Instituto de la Democracia

\section{De votos a escaños}

\section{Los sistemas electorales}

Los sistemas electorales instrumentalizan las reglas del juego para la designación de autoridades de elección popular. De modo restringido, se podría definir a un sistema electoral como el conjunto de normas (sean constitucionales o legales) que se relacionan entre sí y que establecen fundamentalmente dos efectos: la forma cómo los electores, a través

4 En el Ecuador los electores hombres y mujeres votan por separado en sus respectivas juntas receptoras del voto. 
del voto, expresan su preferencia por una organización política o un candidato; y, cómo estos votos se transforman en cargos públicos (presidente, asambleístas, alcalde, concejales, entre otros). Este conjunto de normas responden a la realidad de cada país y su respectivo contexto político, lo que obliga en este punto a afirmar que no existe un sistema electoral, como tal, por el contrario debemos referirnos necesariamente a sistemas electorales.

Desde esta premisa, los efectos de los sistemas electorales han sido rigurosa y sistemáticamente estudiados desde la ciencia política hasta llegar a un consenso que determina que, para estudiar estos sistemas, es primordial comprender la naturaleza de los componentes que lo conforman; es más, "los efectos políticos de los sistemas electorales en su conjunto dependen de la combinación de sus respectivos componentes singulares, los cuales podrían verse reforzados, atenuados o neutralizados dependiendo de su diseño concreto" (Nohlen, 2013: 7).

La mejor aproximación teórica sobre los componentes constitutivos de los sistemas electorales la presentan Nohlen (2007; 2013), Lijphart (1994) y Sartori (1986; 2012), y se puede clasificar en cuatro: 1) el tipo y tamańo de las circunscripciones electorales; 2) las formas de candidatura y de votación; 3) la conversión de votos en escańos; y 4) las barreras legales. Al primero se lo puede resumir como "el proceso de determinación del número y tamaño de la unidad territorial en la cual los votos emitidos por los electores constituyen el fundamento para la repartición de escańos" (Rae, 1971: 19). El segundo se refiere, por un lado, a la forma cómo las listas o sus respectivos candidatos se presentan en la papeleta electoral y, por otro lado, a la forma cómo el elector raya su voto en la papeleta electoral. El tercer componente establece el tipo de fórmula o regla de decisión aplicada para convertir los votos de los electores en ganadores de cargos públicos. Finalmente, el cuarto componente instituye los tipos de barreras legales o porcentajes mínimos de votación que las organizaciones políticas deben obtener para ser consideradas en la repartición de escaños en circunscripciones pluripersonales o nacional. 
Los sistemas electorales, en este sentido, desempeñan indudablemente un papel fundamental sobre el proceso político ya que, como lo plantea Nohlen, "tienen repercusiones sobre el comportamiento electoral y, de este modo, sobre la votación” (Pachano, 1998: 11). Las características de los sistemas electorales dependen entonces del diseño institucional y de los actores involucrados en este; siguiendo a Colomer (2003), son los partidos los que eligen los sistemas electorales. Esto podría explicar cómo es que en el caso ecuatoriano se ha empleado un sistema electoral distinto para cada elección legislativa, o dicho de otra manera, el diseńo institucional ha pasado por varios reformistas a través de los años, unos con posiciones distintas a otros. La Tabla 2 presenta un recuento de las principales características del sistema electoral del Ecuador entre 1978 hasta el 2013.

\section{Tabla 2.}

Sistema electoral ecuatoriano para las elecciones legislativas y sus reformas

\begin{tabular}{|c|c|c|c|c|c|c|}
\hline Periodo & $\begin{array}{l}\text { Principio de } \\
\text { representación }\end{array}$ & $\begin{array}{l}\text { Fórmula } \\
\text { electoral }\end{array}$ & $\begin{array}{c}\text { Tipo de } \\
\text { legisladores }\end{array}$ & 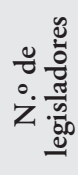 & $\begin{array}{c}\text { Forma de } \\
\text { candidatura y } \\
\text { de votación }\end{array}$ & 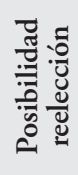 \\
\hline 1978 & Proporcional & $\begin{array}{l}\text { Cociente o } \\
\text { residuo }\end{array}$ & $\begin{array}{l}\text { Nacionales } \\
\text { Provinciales }\end{array}$ & 69 & $\begin{array}{l}\text { Lista cerrada } \\
\text { y bloqueada }\end{array}$ & No \\
\hline 1983 & Proporcional & $\begin{array}{c}\text { Cociente o } \\
\text { residuo }\end{array}$ & $\begin{array}{l}\text { Nacionales } \\
\text { Provinciales }\end{array}$ & 71 & $\begin{array}{l}\text { Lista cerrada } \\
\text { y bloqueada }\end{array}$ & No \\
\hline 1985 & Mayoritario & & $\begin{array}{l}\text { Nacionales } \\
\text { Provinciales }\end{array}$ & 71 & $\begin{array}{l}\text { Lista cerrada } \\
\text { y bloqueada }\end{array}$ & No \\
\hline 1986 & Proporcional & $\begin{array}{l}\text { Cociente o } \\
\text { residuo }\end{array}$ & $\begin{array}{l}\text { Nacionales } \\
\text { Provinciales }\end{array}$ & 71 & $\begin{array}{l}\text { Lista cerrada } \\
\text { y bloqueada }\end{array}$ & No \\
\hline 1994 & Proporcional & $\begin{array}{l}\text { Cociente o } \\
\text { residuo }\end{array}$ & $\begin{array}{l}\text { Nacionales } \\
\text { Provinciales }\end{array}$ & 77 & $\begin{array}{l}\text { Lista cerrada } \\
\text { y bloqueada }\end{array}$ & Sí \\
\hline 1996 & Proporcional & $\begin{array}{l}\text { Cociente o } \\
\text { residuo }\end{array}$ & $\begin{array}{l}\text { Nacionales } \\
\text { Provinciales }\end{array}$ & 82 & $\begin{array}{l}\text { Lista cerrada } \\
\text { y bloqueada }\end{array}$ & Sí \\
\hline
\end{tabular}




\begin{tabular}{l|c|c|l|l|c|c}
\hline 1998 & $\begin{array}{c}\text { Proporcional } \\
\text { mixta }\end{array}$ & $\begin{array}{c}\text { Votación } \\
\text { personali- } \\
\text { zada }\end{array}$ & Provinciales & 121 & $\begin{array}{c}\text { Listas abier- } \\
\text { tas con voto } \\
\text { personalizado } \\
\text { múltiple }\end{array}$ & Sí \\
\hline 2000 & Proporcional & D'Hondt & Provinciales & 100 & $\begin{array}{c}\text { Listas abier- } \\
\text { tas con voto } \\
\text { personalizado } \\
\text { múltiple }\end{array}$ & Sí \\
\hline 2003 & Proporcional & $\begin{array}{c}\text { Imperiali } \\
\text { (corregido) }\end{array}$ & Provinciales & 100 & $\begin{array}{c}\text { Listas abier- } \\
\text { tas con voto } \\
\text { personalizado } \\
\text { múltiple }\end{array}$ & Sí \\
\hline 2013 & Proporcional & D'Hondt & Provinciales & 137 & $\begin{array}{c}\text { Listas abier- } \\
\text { tas con voto } \\
\text { personalizado } \\
\text { múltiple }\end{array}$ & Sí \\
\hline \multirow{2}{*}{2009} & Proporcional & Webster & $\begin{array}{c}\text { Nacionales } \\
\text { provinciales }\end{array}$ & 124 & $\begin{array}{c}\text { Listas abier- } \\
\text { tas con voto } \\
\text { personalizado } \\
\text { múltiple }\end{array}$ & Sín \\
\hline
\end{tabular}

Fuente: Freidenberg (2008: 40). Actualización propia

\section{Tipo y tamaño de las circunscripciones electorales}

Se conoce como tipo de circunscripción a la naturaleza espacial del territorio donde se va a elegir una dignidad de elección popular, mientras que el tamaño se refiere al número de escaños que se eligen en determinada circunscripción (Instituto de la Democracia, 2012). Junto a la fórmula de asignación de escaños, la circunscripción electoral es el componente más relevante de un sistema electoral, debido a que su diseño establece la relación entre la población y el número de representantes a 
elegir. Es así que entre los efectos más importantes que arroja este componente está la proporción o desproporción de dignidades que se eligen en cada circunscripción. En ciertos casos, el número de escaños a elegir es proporcional a la población de determinada circunscripción mientras que en otros casos el efecto es todo lo contrario.

El Código de la Democracia (Art. 150, 151, 154, 155, 158) establece varios tipos de circunscripciones de acuerdo a la dignidad a elegir. Para la dignidad de presidente, vicepresidente y asambleístas nacionales, la circunscripción es de tipo nacional, debido a que se considera todo el territorio nacional como una unidad territorial. Empero, para la elección de asambleístas provinciales, distritales y especiales del exterior, el tipo de circunscripción responde a su respectiva unidad territorial, por ejemplo, para la elección de asambleístas de la provincia de Pichincha, la circunscripción electoral es el territorio de la provincia de Pichincha.

Sobre el tamaño, como se mencionó anteriormente, éste no hace referencia a la extensión territorial, sino al número de escaños que se adjudican en la circunscripción. Para el caso de las elecciones legislativas en el Ecuador, el tamańo de las circunscripciones electorales son de orden plurinominal, lo que quiere decir, circunscripciones donde se elige más de un candidato en cada unidad territorial. Las circunscripciones plurinominales pueden diferenciarse, a su vez, en pequeñas (circunscripciones que eligen de dos a cinco escaños), medianas (circunscripciones que eligen de seis a diez escaños) y grandes (con más de diez escaños a elegir). Para las elecciones legislativas del 17 de febrero de 2013 hubieron treinta y tres circunscripciones pequeñas de un total de treinta y cinco; nueve de ellas eligieron dos escańos, en seis se eligieron tres escańos, diez circunscripciones eligieron cuatro escańos y en ocho circunscripciones cinco escaños; de las dos restantes, una fue mediana, con seis escańos, y una fue grande con quince escaños (Ver Tabla 3). Esto es crucial debido a que el tamaño de la circunscripción es decisivo para los efectos proporcionales de un sistema electoral (Cisneros, 2013: 5); en otras palabras, mientras más pequeña es la circunscripción menos proporcional es el resultado. 
Tabla 3.

Clasificación de las circunscripciones para la elección de Asamblea Nacional 2013 según su tamaño

\begin{tabular}{|c|c|c|c|c|}
\hline $\begin{array}{l}\text { Tamaño de } \\
\text { la circuns- } \\
\text { cripción }\end{array}$ & $\begin{array}{l}\text { Número total } \\
\text { de circuns- } \\
\text { cripciones }\end{array}$ & $\begin{array}{l}\text { Unidades terri- } \\
\text { toriales de cada } \\
\text { circunscripción }\end{array}$ & $\begin{array}{l}\text { Total de asam- } \\
\text { bleístas por } \\
\text { tamaño de cir- } \\
\text { cunscripción }\end{array}$ & $\begin{array}{l}\text { \% de asambleís- } \\
\text { tas por tamaño } \\
\text { de circunscrip- } \\
\text { ción }\end{array}$ \\
\hline 2 escaños & 9 & $\begin{array}{l}\text { Galápagos, Mo- } \\
\text { rona Santiago, } \\
\text { Napo, Orellana, } \\
\text { Pastaza y Zamo- } \\
\text { ra Chinchipe, y } \\
\text { tres circunscrip- } \\
\text { ciones especiales } \\
\text { del exterior } \\
\text { (Estados Unidos } \\
\text { y Canadá; Euro- } \\
\text { pa, Asia y Ocea- } \\
\text { nía; y América } \\
\text { Latina, el Caribe } \\
\text { y África) }\end{array}$ & 18 & $25,7 \%$ \\
\hline 3 escaños & 6 & $\begin{array}{c}\text { Bolívar, Cañar, } \\
\text { Carchi, Santa } \\
\text { Elena, Sucum- } \\
\text { bíos, y una de } \\
\text { la división de } \\
\text { Pichincha }\end{array}$ & 18 & $17 \%$ \\
\hline 4 escaños & 10 & $\begin{array}{l}\text { Chimborazo, } \\
\text { Cotopaxi, } \\
\text { Esmeraldas, } \\
\text { Imbabura, } \\
\text { Loja, Santo } \\
\text { Domingo, Tun- } \\
\text { gurahua, dos de } \\
\text { la división de } \\
\text { Pichincha y una } \\
\text { de la división de } \\
\text { Manabí }\end{array}$ & 40 & $28,6 \%$ \\
\hline
\end{tabular}




\begin{tabular}{|c|c|c|c|c|}
\hline 5 escaños & 8 & $\begin{array}{c}\text { Azuay, El Oro, } \\
1 \text { de la división } \\
\text { de Manabí, } 1 \\
\text { de la división de } \\
\text { Pichincha y } 4 \\
\text { de la división de } \\
\text { Guayas }\end{array}$ & 40 & $22,9 \%$ \\
\hline 6 escaños & 1 & Los Ríos & 6 & $2,9 \%$ \\
\hline 15 escańos & 1 & $\begin{array}{l}\text { Territorio nacio- } \\
\text { nal y especiales } \\
\text { del exterior }\end{array}$ & 15 & $2,9 \%$ \\
\hline $\begin{array}{l}\text { Promedio: } \\
\text { 3,91 } \\
\text { escaños }\end{array}$ & 35 & $\begin{array}{l}1 \text { nacional, } 24 \\
\text { provinciales ( } 3 \\
\text { con subdivi- } \\
\text { sión), } 3 \text { especia- } \\
\text { les del exterior }\end{array}$ & 137 & $100 \%$ \\
\hline
\end{tabular}

Elaboración: Instituto de la Democracia

Más allá del tamaño de la circunscripción, sea esta pequeña, mediana o grande, también es importante considerar el encabezamiento de las listas pluripersonales (si el primer casillero lo ocupa una mujer o un hombre). En países donde las listas pluripersonales reconocen la paridad y la alternabilidad entre mujeres y hombres, como en el caso ecuatoriano, el tamaño de la circunscripción y el encabezamiento de las listas por mujeres u hombres significan una desproporción adicional considerable al momento de asignar los escaños ganadores. Aunque este efecto está estrechamente atado a la composición de las candidaturas de las listas pluripersonales, es preciso aquí considerar la relación entre el tamaño de las circunscripciones electorales y las formas de candidatura como dos componentes de los sistemas electorales que interactúan entre sí. 


\section{Las formas de candidatura y de votación}

Aunque los sistemas electorales dependen necesariamente de la interrelación de sus componentes, las formas de candidatura y de votación sugieren una atención particular, sobre todo por tratarse del componente que permite al elector expresar su preferencia al momento del sufragio. Si bien es cierto que este componente no ejerce, por sí solo, una influencia directa sobre la representación legislativa, como sí lo hacen el tipo y el tamańo de las circunscripciones electorales dentro de la fórmula de conversión de votos en escaños y las barreras legales, su análisis es importante puesto que las formas de candidaturas y votación "inciden en el vínculo elector-candidato y entre éste y su organización política” (Archenti y Tula, 2007: 190), es decir, este componente impone significativas repercusiones sobre el comportamiento electoral.

De acuerdo con Nohlen (2013), las formas de candidaturas están íntimamente relacionadas con la forma de votación debido a que la primera dispone la forma cómo los candidatos se presentan en la papeleta y, por tanto, cómo el elector debe decidir sus preferencias, mientras que la segunda determina la forma cómo el elector endosa su preferencia, ya sea hacia una o varias organizaciones políticas o hacia sus respectivos candidatos.

Las formas de candidatura y de votación se manifiestan en todos los sistemas electorales con uno de los siguientes tipos de listas: las listas bloqueadas o aquellas que permiten al elector emitir su voto por una sola lista o en plancha; las listas cerradas y no bloqueadas las cuales permiten al elector alterar el orden de ubicación de los candidatos dentro de una sola lista; y las listas abiertas caracterizadas por brindarle al elector la posibilidad de escoger sus candidatos entre varias listas.

Las listas abiertas en el contexto ecuatoriano merecen especial atención ${ }^{5}$. El sistema de listas abiertas (Ver Tabla 2) con voto personali-

5 Para la elección de la Asamblea Nacional y el Parlamento Andino el sistema aplicó la forma de candidatura de listas abiertas establecida en el Artículo 160 del Código de la Democracia (2009). La forma de votación, por su parte, entendida como la manera en que el elector raya su voto en la papeleta electoral en elecciones pluripersonales se establece en el Artículo 120 del Código de la Democracia. 
zado múltiple es un componente que aparece en el Ecuador a partir del plebiscito de mayo de 1997 como alternativa a las listas cerradas y bloqueadas. Esta forma de votación se ha mantenido vigente hasta la actualidad a pesar de que nace por una mala interpretación, ya que la pregunta formulada en 1997, “en forma equívoca y poco clara” llevó a un sistema personalizado de listas abiertas, en lugar de la instalación de un sistema de listas cerradas y no bloqueadas (Pachano, 2007: 68).

En general, la modalidad de votación de listas abiertas ha generado varios argumentos a favor y en contra, principalmente por tratarse de una forma de votación que permite al elector decidir por dos o más opciones entre varias líneas partidistas. Entre los argumentos a favor están los de los propios reformistas del diseño electoral en países donde se aplica este sistema, que, como se mencionó anteriormente, son los mismos partidos políticos. De igual forma hay quienes defienden los sistemas de listas abiertas con voto personalizado; en este caso, la reputación que adquieren legisladores que se ubicaron en listas abiertas es considerablemente mayor a aquellos legisladores que lograron escańos en listas cerradas (Carey y Shugart, 1995; Taagapera y Shugart, 1989).

Entre los argumentos en contra destacan aquellas posiciones que reiteran el prejuicio que resulta votar entre listas por cuanto el elector incurre en otorgar su voto a varias líneas partidistas e ideológicas, muchas de ellas de corte opuesto; así mismo, "el emitir sus preferencias a varios candidatos entre varias listas conlleva a efectos no deseados del diseño institucional sobre el sistema de partidos" (Freidenberg, 2008: 44); por ejemplo, el solo hecho de votar entre listas conduce inclusive a que "antes del cómputo de los votos", el mismo elector coadyuve a la fragmentación partidaria en el Legislativo (Pachano, 2007: 170).

\section{Las fórmulas de conversión de votos en escaños}

De manera general, es el procedimiento mediante el cual se aplica una fórmula matemática para convertir los votos en escaños después del 
conteo de votos para así determinar qué organizaciones políticas acceden a las dignidades de elección popular de orden pluripersonal; de ahí el porqué este es uno de los componentes más relevantes de un sistema electoral.

Las fórmulas responden a dos criterios de asignación: la regla de decisión por mayoría y las reglas de proporcionalidad (Lijphart, 1994). El primero es un criterio que adopta lógicas de decisión por mayoría absoluta o mayoría relativa; el segundo responde al criterio que otorga como ganadores a quienes han alcanzado cierto porcentaje en relación al total de votos obtenidos y al tamaño de la circunscripción electoral.

En el Ecuador, el sistema electoral legislativo es muy complejo, la regla de decisión es mixta, es decir, adopta principios de ambas reglas. Por un lado, se aplica el principio de proporcionalidad para determinar el número de escaños que le corresponden a cada organización política a través de una fórmula de divisores impares o método Webster para la asignación de asambleístas nacionales, y una fórmula de divisores continuos o método D'Hondt para la asignación del resto de escaños legislativos ${ }^{6}$. Por otro lado, se aplica el principio de mayoría para determinar los ganadores dentro de cada lista. Es decir, "una vez que se conoce el número de escaños que se adjudicarán a las organizaciones políticas ganadoras, son los candidatos más votados dentro de cada lista quienes acceden a ocupar dichos escaños, independientemente del orden presentado en la papeleta" (Instituto de la Democracia, 2012: 9). Para las elecciones unipersonales, sin embargo, el sistema combina la regla de decisión de mayoría relativa con una exigencia adicional, tanto en el porcentaje de votos obtenidos como de la diferencia entre la votación de los dos candidatos más votados ${ }^{7}$.

6 Tanto la fórmula de divisores impares como la de divisores continuos se establecen en el Artículo 164 del Código de la Democracia (2009).

7 Artículo 143 de la Constitución de la República (2008) y el Artículo 89 del Código de la Democracia (2009). A esta fórmula se la denomina mayoría relativa calificada, la cual designa como ganador al binomio que haya obtenido en la primera vuelta al menos el $40 \%$ de los votos válidos emitidos y una diferencia mayor de diez puntos porcentuales sobre la votación alcanzada por el binomio ubicado en el segundo lugar, o al binomio que haya alcanzado la mayoría absoluta del total de votos válidos emitidos, es decir, más del 50\% de los votos a su favor. En el caso de no 


\section{Las barreras legales}

En cuanto a las barreras legales, la ley electoral ecuatoriana no contempla una barrera o porcentaje de votos mínimos para que una organización política sea tomada en cuenta en la asignación de escańos para dignidades de elección pluripersonal. Este es el único componente, de acuerdo a la clasificación teórica, que no forma parte del sistema electoral ecuatoriano ${ }^{8}$.

\section{Las cuotas de género}

A pesar que las cuotas de género no se establecen como uno de los componentes de los sistemas electorales, estas disposiciones normativas resultan ser decisivas en la composición final de cuerpos colegiados.

Es así que los sistemas electorales, en especial la forma de votación personalizada múltiple, ocupan un importante espacio en el tratamiento de estudios de caso ligados a la participación electoral de poblaciones tradicionalmente excluidas, entre las cuales se destaca esencialmente la participación de las mujeres (Rule, 1987; Archenti y Tula, 2007; Meier, 2008; de Souza, 2013). A esta rama del debate se le ha acuñado el nombre de ley de cuotas, sistema de cuotas, cuotas de género, entre otros.

En el marco de la participación e inclusión de la ciudadanía como candidatos para elecciones a cargos de representación popular, las cuotas de género han recuperado un importante espacio en el debate internacional, sobre todo en el ámbito legislativo; más aún, cuando este ámbito es por naturaleza sinónimo de representación y de deliberación

haber logrado estas dos primeras condiciones, el sistema obliga una segunda vuelta electoral con los dos binomios más votados en la primera vuelta, cuestión que no sucedió en las elecciones para presidente y vicepresidente del ańo 2013.

8 La ley sí contempla una barrera legal, pero no como uno de los cuatro componentes de un sistema electoral sino como el porcentaje de votación mínima requerida para que una organización política pueda conservar su registro electoral, que en el caso ecuatoriano es el $4 \%$ de los votos válidos en dos elecciones pluripersonales consecutivas a nivel nacional, o en su efecto tres representantes a la Asamblea Nacional. Así lo establece el numeral 3 del Artículo 327 del Código de la Democracia (2009). 
política. En un estudio realizado por el Instituto Federal Electoral de México, de una muestra de dieciocho legislaturas, trece de ellas consagran disposiciones expresas en esta materia (cfr. IFE, 2011: 54).

El Ecuador se encuentra en el grupo de los trece casos y, aunque la actual Constitución exige la aplicación de los principios de paridad y alternabilidad para la conformación de todas las listas pluripersonales, este no siempre fue el caso. Por ejemplo, desde 1979 hasta 1997 la ley no establecía ningún tipo de cuota. En 1998, la cuota pasó a ser del 30\% de participación femenina para las candidaturas a dignidades de elección pluripersonal con un incremento progresivo, de acuerdo a la ley electoral, hasta alcanzar el 50\% de representación en las candidaturas (Pachano, 2007); en este periodo no se estableció la obligatoriedad del orden de ubicación de las mujeres en determinados puestos de la lista. Con la Constitución del año 2008 se establece un sistema electoral conforme a los principios de paridad y alternabilidad entre mujeres y hombres para las candidaturas de elecciones pluripersonales (Art. 116), con esto se aseguró al menos la posibilidad para que tanto mujeres como hombres integren sus respectivas listas conforme a estos principios?

\section{Particularidades del sistema ecuatoriano}

En resumen, la manera cómo se manifiestan los componentes del sistema electoral ecuatoriano hacen de este un sistema muy particular al resto de sistemas electorales de países vecinos. Por ejemplo, mientras que en Perú se dispone la aplicación de barreras legales, en el Ecuador este componente no se establece en la norma; entretanto en Brasil se utiliza una sola fórmula de asignación de escaños legislativos (cociente o Hare), en el Ecuador se aplican dos fórmulas (Webster y $D^{\prime}$ Hondt); mientras que en Paraguay las listas son bloqueadas, en el Ecuador son

9 Cabe indicar que los principios de paridad y alternabilidad son solo requisitos para la inscripción de las listas pluripersonales y no así condicionalidad para la conformación de cuerpos colegiados. Esto contesta la pregunta del porqué, a pesar de contar con listas que respetan estos principios, la composición de la Asamblea Nacional, por ejemplo, no refleja el mismo porcentaje. 
abiertas; o cuando en Argentina no se permite el voto preferente, en el Ecuador se emplea el voto preferencial múltiple (IFE, 2011).

\section{Análisis: el caso de Pichincha}

Se dijo que los sistemas electorales establecen fundamentalmente dos efectos: la forma cómo los electores, a través del voto, expresan su preferencia por una organización política o un candidato y, cómo estos votos se transforman en cargos públicos. Los resultados electorales 2013, sobre la base de la muestra recogida por la Delegación Electoral Provincial de la provincia de Pichincha, permiten observar dichos efectos. Además, los resultados también permiten ver un efecto adicional: la composición de la Asamblea Nacional conforme a la aplicación de los principios de paridad y alternabilidad. A continuación se detallan los tres efectos por separado.

\section{Primer efecto: la expresión de las preferencias a través del voto}

De hecho en el Ecuador, la forma cómo el elector expresa sus preferencias a través del voto está cercanamente relacionada a las listas abiertas y al voto personalizado múltiple; este último es muy particular, no solo por tratarse de una forma de votación poco aplicada en países de la región sino también por tratarse de un mecanismo que permite de forma expresa que el elector divida o fraccione su voto. Dicho de otra manera, el permitir que la preferencia de un elector pueda ser distribuida por uno, algunos o todos los candidatos de una sola lista, o por algunos o todos los candidatos entre distintas listas hasta completar el número de escaños permitido para la dignidad a elegir, determina que un voto se divida en varias fracciones de voto.

En sistemas electorales donde se aplican las listas cerradas y donde no se contempla la modalidad del voto preferente, como es el caso de 
Uruguay por ejemplo, la preferencia del elector se constituye en un solo voto para todos los candidatos dentro de una sola lista pluripersonal. Sin embargo en el Ecuador y tomando como ejemplo el caso de la provincia de Pichincha, en donde se eligieron dieciséis asambleístas en cuatro circunscripciones electorales, un voto es el resultado del total de votos nominales utilizados por el elector, lo que quiere decir que si un elector por ejemplo optó por señalar tres de los cuatro casilleros en una sola lista o entre listas, éste utilizó solamente el 0,75 de su voto. Para asignar escańos legislativos en el Ecuador, se debe sumar necesariamente los votos nominales (los votos individuales) de cada uno de los candidatos y candidatas para obtener el total de votos nominales de cada organización política, previo a la aplicación de la fórmula de asignación de escańos respectiva.

Si bien es válida la tesis de que la opción del voto preferencial múltiple "amplia la posibilidad de relación entre el elector y sus candidatos" (Nohlen, 2007; 301) al permitir que el primero señale sus preferencias indistintamente de la afiliación política e ideológica de sus candidatos preferidos y, por tanto, abrir el universo de posibilidades para que el elector seleccione democráticamente las y los mejores candidatos, ¿qué representa en términos reales la opción del voto preferencial múltiple versus la opción del voto por todos los candidatos en una sola lista? Por un lado, como ya se indicó anteriormente, el fraccionar el voto en varios votos entre distintas listas implica un efecto contraproducente, ya que, además de la posibilidad que tiene el elector de escoger en la misma papeleta a candidatos pertenecientes a organizaciones políticas similares o antagónicas -acarreando con esto evidentes repercusiones-, ello también se constituye en un enorme estímulo para la fragmentación (Pachano, 2007). Empero, el resultado efectivo de facultar al elector la posibilidad de elegir entre listas demuestra el poco interés del electorado en optar por esta forma de votación; al menos, la evidencia estadística así lo demuestra. Del total de papeletas válidas de la muestra de Pichincha se obtiene un considerable 79,52\% de electores que prefieren votar por todos los candidatos de una sola lista, frente a un 
20,48\% de electores que prefieren votar entre listas (Ver Tabla 4), de lo que, en términos generales, se podría entrever que el voto por una sola lista representa un voto de confianza a la organización política más que a las candidaturas en forma individual.

\section{Tabla 4.}

Votación por una sola lista y entre listas en la provincia de Pichincha

\begin{tabular}{c|c|c|c|c|c}
\hline $\begin{array}{c}\text { Dignidades a } \\
\text { elegir }\end{array}$ & $\begin{array}{c}\text { Votación por } \\
\text { una sola lista }\end{array}$ & $\%$ & $\begin{array}{c}\text { Votación } \\
\text { entre } \\
\text { listas }\end{array}$ & $\%$ & $\begin{array}{c}\text { Total papeletas } \\
\text { válidas de la } \\
\text { muestra }\end{array}$ \\
\hline $\begin{array}{c}\text { Asambleístas } \\
\text { nacionales }\end{array}$ & 87127 & 77,15 & 25809 & 22,85 & 112936 \\
\hline $\begin{array}{c}\text { Asambleístas } \\
\text { provinciales }\end{array}$ & 88681 & 78,81 & 23841 & 21,19 & 112522 \\
\hline $\begin{array}{c}\text { Parlamentarios } \\
\text { andinos }\end{array}$ & 89758 & 82,72 & 18744 & 17,28 & 108502 \\
\hline $\begin{array}{c}\text { Total papele- } \\
\text { tas válidas de } \\
\text { la muestra }\end{array}$ & 265566 & 79,52 & 68394 & 20,48 & 333960 \\
\hline
\end{tabular}

Nota: Votación en relación al total de papeletas válidas de la muestra para cada dignidad

Fuente: Delegación Electoral Provincial de Pichincha (2013)

Elaboración: Instituto de la Democracia

Es más, para aquellos electores que no optaron por usar el total de opciones para los cargos a elegir, el 69,9\% prefirió votar por candidatos de una misma lista en lugar de distribuir su voto entre varias listas. Situación similar se observa en la elección de parlamentarios andinos en donde el $84,5 \%$ de electores que no votaron por todos los candidatos de una sola lista, sí lo hicieron por candidatos de una sola lista (Ver Gráfico 1).

Siguiendo los datos, en la muestra de papeletas para asambleístas nacionales la segunda mejor opción fue escoger entre dos organizaciones políticas. La opción de escoger entre once organizaciones políticas no 
fue la menor de todas las opciones; mientras que escoger entre nueve o diez organizaciones políticas fueron las opciones menos utilizadas. Con respecto a las papeletas para parlamentarios andinos, la segunda mejor opción también fue escoger entre dos organizaciones políticas; la opción de escoger entre cuatro organizaciones políticas no fue la menor de todas las opciones; mientras que la opción de escoger entre tres organizaciones políticas fue la opción menos utilizada.

\section{Gráfico 1.}

Electores que optaron por no usar el total de opciones de voto

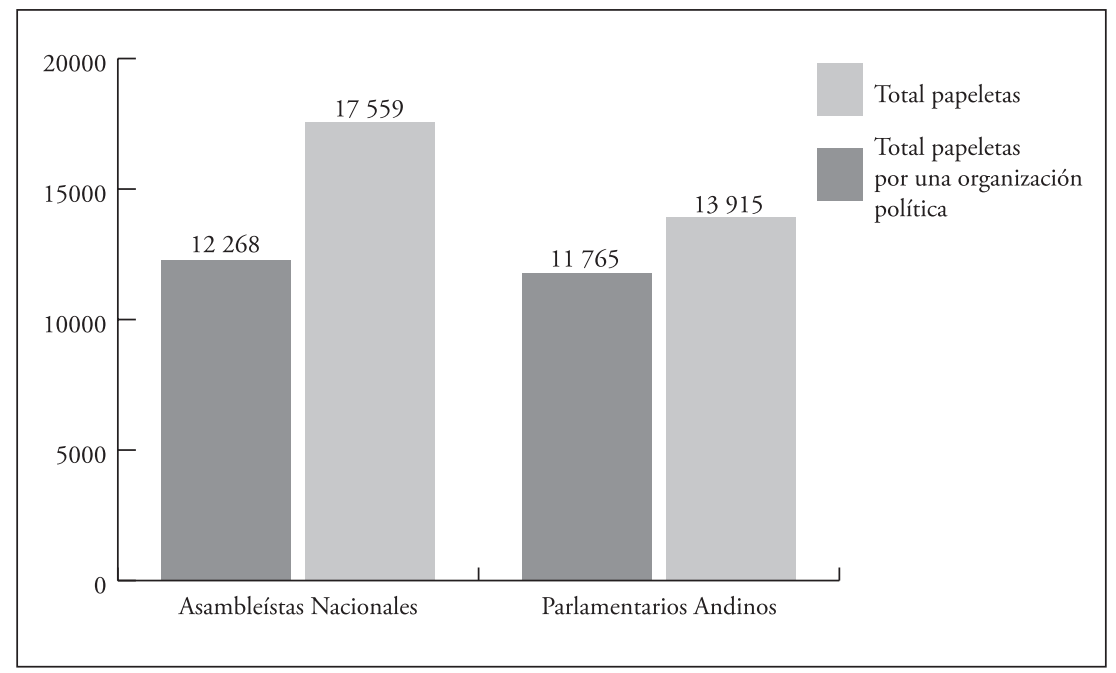

Fuente: Delegación Electoral Provincial de Pichincha (2013)

Elaboración: Instituto de la Democracia

Uno de los factores más importantes que contribuyen al alto porcentaje de votación por una sola lista es la preferencia partidista del electorado hacia a una organización política determinada. En el caso de Pichincha, los resultados de asambleístas para las cuatro circunscripciones electorales de la provincia muestran una alta votación para los candidatos del movimiento político PAIS, tanto así que de los dieciséis escańos asig- 
nados, trece fueron adjudicados a esta organización política (Ver Tabla 5), por tanto, el alto porcentaje de votación por una sola lista versus la opción de votación entre listas tiene efectos decisivos y determinantes a la adjudicación de los escaños ganadores.

\section{Tabla 5.}

Resultados electorales para asambleístas provinciales de Pichincha

\begin{tabular}{c|c|c|c}
\hline Asambleístas electos & $\begin{array}{c}\text { Organización } \\
\text { política }\end{array}$ & Votación nominal & $\begin{array}{c}\text { Suma de votos } \\
\text { nominales }\end{array}$ \\
\hline \multicolumn{5}{c}{ Circunscripción 1 } \\
\hline Francisco Velasco & PAIS & 218866 & 830026 \\
\hline Ximena Ponce & PAIS & 204771 & 830026 \\
\hline Fernando Bustamante & PAIS & 206014 & 830026 \\
\hline Andrés Páez & CREO & 115337 & 381106 \\
\hline \multicolumn{5}{|c|}{ Circunscripción 2 } \\
\hline Virgilio Hernández & PAIS & 264085 & 1237342 \\
\hline Paola Pabón & PAIS & 250506 & 1237343 \\
\hline Fausto Cayambe & PAIS & 242762 & 1237344 \\
\hline María José Carrión & PAIS & 240913 & 1237345 \\
\hline Wilson Chicaiza & CREO & 56571 & 261284 \\
\hline \multicolumn{5}{|c|}{ Circunscripción 3 } \\
\hline María Augusta Calle & PAIS & 175786 & 646922 \\
\hline Fausto Terán Sarzosa & PAIS & 157083 & 646922 \\
\hline Soledad Buendía & PAIS & 159487 & 646922 \\
\hline Diego Salgado & CREO & 45388 & 167853 \\
\hline
\end{tabular}




\begin{tabular}{c|c|c|c}
\hline \multicolumn{4}{c}{ Circunscripción 4 } \\
\hline Mauricio Proaño & PAIS & 86729 & 241490 \\
\hline Esthela Acero & PAIS & 79120 & 241490 \\
\hline Nelson Serrano & PAIS & 75641 & 241490 \\
\hline
\end{tabular}

Fuente: CNE (2013). Elaboración propia

De igual forma, otros factores podrían estar aportando al alto porcentaje de votación por una sola lista. Podría decirse que uno de ellos es el conocimiento pleno del elector sobre el sistema electoral vigente debido a que el voto para todas las candidatas y candidatos de una sola lista amplia la posibilidad de que estas candidaturas obtengan escaños legislativos versus a aquellos votos entre listas. En este sentido, si bien es cierto que para asignar escańos es necesario, como primer paso, sumar el total de votos nominales de cada uno de los candidatos, las fórmulas de asignación de escaños solo se aplican a la suma total de los votos nominales de todos los candidatos dentro de una lista, lo que quiere decir que mientras más votos se emitan a una sola lista, mayor la posibilidad que esa lista gane escaños. Habría que preguntar al elector si en efecto conoce o no cómo las fórmulas asignan escaños para confirmar o descartar esta hipótesis.

Es necesario indagar las particularidades del voto preferencial múltiple y entender el contexto sobre el cual se manifiestan estas preferencias. A diferencia del voto por una sola lista, el voto entre listas resalta la afinidad de los electores hacia candidaturas individuales entre varias listas, independientemente de la afiliación partidaria de estos candidatos. Si bien es cierto que el porcentaje de votación entre listas es significativamente menor al voto para una sola lista, y que además, por sí solo, no representa un efecto decidor para la asignación de escańos, no deja de ser una expresión democrática válida. A continuación se detalla la votación entre listas en todos los cantones de la provincia. 
Tabla 6.

Porcentaje de votación entre listas en la provincia de Pichincha

\begin{tabular}{l|c|c|c}
\hline Cantón & $\begin{array}{c}\text { Asambleístas } \\
\text { nacionales }\end{array}$ & $\begin{array}{c}\text { Asambleístas } \\
\text { provinciales }\end{array}$ & $\begin{array}{c}\text { Parlamentarios } \\
\text { andinos }\end{array}$ \\
\hline Cayambe & 30,65 & 33,35 & 29,23 \\
\hline Mejía & 26,77 & 30,27 & 24,15 \\
\hline Pedro Moncayo & 32,72 & 34,39 & 27,06 \\
\hline $\begin{array}{l}\text { Pedro Vicente } \\
\text { Maldonado }\end{array}$ & 37,60 & 39,03 & 31,50 \\
\hline Puerto Quito & 39,44 & 45,39 & 35,99 \\
\hline Quito & 21,71 & 19,17 & 15,61 \\
\hline Rumiñahui & 21,43 & 20,04 & 15,46 \\
\hline San Miguel de los Bancos & 38,87 & 42,68 & 35,23 \\
\hline Total & 22,85 & 21,19 & 17,28 \\
\hline
\end{tabular}

Nota: Porcentaje en relación al total de papeletas válidas de la muestra por cantón para cada dignidad Fuente: Delegación Electoral Provincial de Pichincha (2013)

Elaboración: Instituto de la Democracia

Asimismo, el efecto más influyente de la votación preferencial múltiple para el caso ecuatoriano es su contribución a la suma total de votos nominales para cada organización política y, por consiguiente, a la adjudicación final de los escańos ganadores. Cabe recordar que la asignación final de escaños se otorga a los candidatos más votados de la lista y, justamente, la votación preferencial múltiple da la posibilidad que unos candidatos obtengan mayor número de votos que otros, y por lo tanto que sean los ganadores independientemente del orden de ubicación en la lista. En los resultados de la elección de asambleístas nacionales se pudo observar este efecto (Ver Tabla 7). 
Tabla 7.

Resultados electorales para asambleístas nacionales

\begin{tabular}{|c|c|c|c|c|}
\hline Asambleísta & $\begin{array}{l}\text { Organización } \\
\text { política }\end{array}$ & $\begin{array}{l}\text { Votación } \\
\text { nominal }\end{array}$ & $\begin{array}{c}\text { Suma de votos } \\
\text { nominales }\end{array}$ & $\begin{array}{l}\text { Ubicación } \\
\text { en la lista }\end{array}$ \\
\hline Gabriela Rivadeneira & PAIS & 3498379 & 45955995 & 1 \\
\hline Fernando Cordero & PAIS & 3224291 & 45955995 & 2 \\
\hline Marcela Aguiñaga & PAIS & 3127267 & 45955995 & 3 \\
\hline Iván Hurtado & PAIS & 3111895 & 45955995 & 8 \\
\hline Miguel Carvajal & PAIS & 3061493 & 45955995 & 4 \\
\hline Carlos Viteri & PAIS & 3059113 & 45955995 & 6 \\
\hline Marllely Vásconez & PAIS & 3056851 & 45955995 & 5 \\
\hline Linda Machuca & PAIS & 3027923 & 45955995 & 7 \\
\hline Mae Montaño & CREO & 835146 & 10032804 & 1 \\
\hline Patricio Donoso & CREO & 712466 & 10032804 & 2 \\
\hline Cynthia Viteri & PSC & 853216 & 7901315 & 1 \\
\hline Gilmar Gutiérrez & PSP & 586319 & 4955320 & 1 \\
\hline Lourdes Tibán & $\begin{array}{l}\text { MUPP- } \\
\text { MPD }\end{array}$ & 494879 & 4151000 & 1 \\
\hline Abdalá Bucaram & PRE & 499428 & 3960188 & 1 \\
\hline Ramiro Aguilar & SUMA & 271609 & 2829034 & 1 \\
\hline
\end{tabular}

Fuente: CNE (2013). Elaboración propia

En cuanto al voto del elector por los candidatos de una sola lista o entre listas, el voto preferencial múltiple también posibilita la elección solamente para los candidatos por un determinado sexo. Sin embargo este no es el caso para los electores de Pichincha. Los electores de esta provincia definen sus preferencias electorales (Ver Tabla 8) tanto para mujeres como para candidatos hombres; solo un minúsculo porcentaje 
de electores hombres prefieren votar solo por candidatos hombres y lo propio se evidencia en la población femenina.

\section{Tabla 8.}

Porcentaje de votación femenina y masculina en Pichincha con base al sexo de los candidatos

\begin{tabular}{|c|c|c|c|c|c|c|c|c|c|}
\hline & \multicolumn{3}{|c|}{ Candidatas mujeres } & \multicolumn{3}{|c|}{ Candidatos hombres } & \multicolumn{3}{|c|}{ Candidaturas mixtas } \\
\hline & 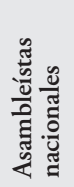 & 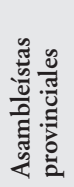 & 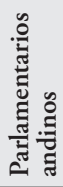 & 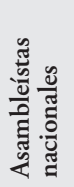 & 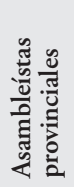 & 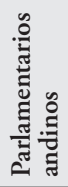 & 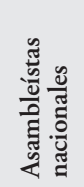 & 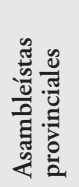 & 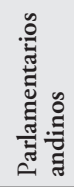 \\
\hline $\begin{array}{l}\text { Electoras } \\
\text { mujeres }\end{array}$ & 4,32 & 3,55 & 1,93 & 3,08 & 7,24 & 7,82 & 77,11 & 73,68 & 70,01 \\
\hline $\begin{array}{l}\text { Electores } \\
\text { hombres }\end{array}$ & 3,47 & 2,89 & 1,51 & 3,27 & 6,77 & 6,79 & 78,12 & 74,95 & 72,69 \\
\hline Promedio & 3,90 & 3,22 & 1,72 & 3,18 & 7,01 & 7,31 & 77,62 & 74,32 & 71,35 \\
\hline
\end{tabular}

Nota: Porcentaje sobre el total de papeletas de la muestra incluyendo votos blancos y nulos Fuente: Delegación Electoral Provincial de Pichincha (2013)

Elaboración: Instituto de la Democracia

Segundo efecto: la conversión de votos en escaños ganadores

Este efecto está estrechamente ligado a la interacción e interrelación del tipo y tamaño de las circunscripciones electorales con las fórmulas de asignación de escaños. Para la elección de asambleístas de la provincia de Pichincha, las circunscripciones entran en la clasificación de pequeñas, ya que de las cuatro circunscripciones en dos se eligen cuatro escaños, en una se elige cinco escańos y en otra más se eligen tres escaños. Como se indicó anteriormente, el tamaño de la circunscripción es decisivo para los efectos proporcionales de un sistema electoral: mientras 
más pequeña es la circunscripción menos proporcional es el resultado. Por su parte, de las dos fórmulas de asignación de escaños que establece la ley, el método de divisores continuos es menos proporcional que el método de divisores impares, ya que, a mayor separación de los divisores respectivos, mayor la posibilidad de garantizar proporcionalidad de votos en escaños: para la elección de asambleístas provinciales la ley dispone la aplicación del método de divisores continuos.

De esta manera, el efecto más notable del método de divisores continuos en circunscripciones electorales pequeñas es la desproporcionalidad de escaños que se genera entre las organizaciones políticas más votadas y aquellas organizaciones políticas con menor número de votos. La proporcionalidad, en este sentido, se entiende como la posibilidad de que las minorías partidistas también puedan ocupar escaños en cuerpos colegiados (Sartori, 2012). Un ejemplo de este efecto se observa en los resultados electorales de la circunscripción cuatro de la provincia de Pichincha donde de los tres escańos disponibles, los tres fueron asignados a la fuerza partidista con el mayor número de votos en esa circunscripción (Ver Tabla 5).

\section{Tercer efecto: afectación a la paridad y alternabilidad}

Se ha expuesto que también es importante considerar los efectos que el encabezamiento de las listas pluripersonales produce sobre los resultados electorales. Es importante analizar el encabezamiento como uno de los efectos que determinan la conversión de votos en escańos paritarios, aun cuando este no sea considerado explícitamente como uno de los componentes constitutivos de los sistemas electorales según la categorización tradicional.

Sin lugar a dudas, las cuotas de género han posibilitado la participación de un mayor número de mujeres candidatas dentro de sus respectivas listas, empero, el sistema electoral, sobre todo el tamaño de la circunscripción y la forma de candidatura en listas abiertas (referente al encabezamiento de mujeres u hombres en sus listas), ha imposibilitado 
un acceso real de la mujer, en términos de paridad y alternancia, en cuerpos colegiados como la Asamblea Nacional.

A pesar de que la Constitución de la República (2008) establece un sistema electoral conforme a los principios de paridad y alternabilidad entre mujeres y hombres para las candidaturas de elecciones pluripersonales, los resultados electorales 2013 muestran todo lo contrario: de los ciento treinta y siete escańos, cincuenta y tres fueron adjudicados a mujeres electas. Dicho en términos porcentuales, el 39\% de escańos fueron para mujeres y el $61 \%$ para hombres.

¿Qué explica esta distorsión entre candidaturas paritarias y escaños paritarios? Si hacemos una revisión minuciosa de la relación entre cómo las organizaciones políticas en el Ecuador seleccionan a sus candidaturas y cómo los electores definen sus preferencias, aún encontramos patrones de diseño del sistema electoral vigente que podrían estar produciendo este efecto. Sobre el encabezamiento, de un total de trescientos treinta y cinco candidatos que ocuparon el primer puesto en las listas pluripersonales para el proceso electoral 2013, solo cincuenta y nueve fueron mujeres, es decir, el 17,61\%; de ellas tan solo veinte ganaron escańos. En otras palabras, de las trescientas treinta y cinco candidaturas que encabezaron las listas a nivel nacional, el 5,97\% de mujeres candidatas ganaron un escańo en la Asamblea Nacional. El solo hecho de determinar de manera paritaria la primera ubicación en las listas pluripersonales, redefiniría la proporción entre el número de escańos y el número de mujeres electas en la Asamblea Nacional.

Si se adiciona a esto el tamaño de la circunscripción electoral, la desproporción es aún mayor; esto significa que mientras más pequeña sea la circunscripción electoral, menor probabilidad que mujeres candidatas accedan a cuerpos colegiados. El sistema electoral ecuatoriano, para las treinta y tres circunscripciones electorales pequeñas (de dos a cinco escaños) que corresponden al 94,2\% del total de escaños legislativos, asignó escańos a cuarenta y cuatro mujeres, lo que corresponde al 37,9\% de la Asamblea Nacional. Ante estos números y realidades, el debate sobre el efecto real de los principios de paridad y alternabilidad a la conformación de cuerpos colegiados ni siquiera empieza. 


\section{Conclusiones y recomendaciones}

La primera conclusión refuerza la evidencia empírica sobre la complejidad de los sistemas electorales en torno a la conversión de votos en escaños y la necesaria interrelación entre sus componentes. Es insuficiente analizar los efectos de los sistemas electorales sobre la base de los efectos singulares que producen sus componentes por separado. La segunda conclusión resulta de la significancia de la interrelación entre componentes y trasciende al manifestar que, para medir los efectos de un sistema electoral, no solo es necesario mirar hacia cuatro componentes, $\mathrm{o}$ al menos hacia los que tradicionalmente ha establecido la teoría; sino también, hacia aquellos elementos que participan del sistema y, por consiguiente, afectan a sus resultados; entre ellos está la ley de cuotas y el encabezamiento de mujeres u hombres en las listas. La tercera conclusión confirma la peculiaridad del diseño del sistema electoral ecuatoriano, sobre todo un diseńo que todavía parecería encontrarse en fase de prueba y sobre el cual se le permite al elector, de forma preferencial y múltiple, definir y distribuir sus preferencias; a esto se le suman las fórmulas electorales vigentes - una con efectos proporcionales disímiles a la otra- que se aplican para la asignación de escaños diferentes entre asambleístas nacionales y el resto de asambleístas, aún cuando estos conformen la misma Asamblea Nacional. La complejidad del sistema electoral en el Ecuador obliga a entender y, de esta manera, observar muy de cerca el alcance de los efectos que este produce.

Pero, la conclusión principal va más allá de los efectos que producen sus componentes e invita a tener en cuenta la importancia que representan los sistemas electorales a la posibilidad real de que ciudadanas y ciudadanos, mujeres y hombres, participen en elecciones y no solo eso, sino que el mismo sistema garantice su acceso de forma incluyente y afirmativa a cuerpos colegiados tanto a nivel local como a nivel nacional. Los números advierten señales muy claras de exclusión e imposibilidad de acceso, mientras se mantenga este patrón instrumental, las figuras políticas masculinas seguirán ocupando escaños en las siguientes 
Asambleas Nacionales en una proporción desigual sobre sus pares femeninos, aún cuando la ley disponga la paridad y la alternabilidad como dos condiciones para la participación en elecciones pluripersonales.

En suma, mientras más pequeña es la circunscripción menos proporcional es el resultado; a mayor separación de los divisores de las fórmulas electorales, mayor la posibilidad de garantizar proporcionalidad de votos en escańos; mientras las preferencias del electorado se concentren en una sola lista, mayor la probabilidad que la fuerza partidista con mayor número de votos gane escańos; y a mayor número de candidatas mujeres ocupando el primer lugar en sus respectivas listas, mayor es la probabilidad de que ellas accedan a ocupar cargos públicos. Claro está, la interacción e interrelación entre todos estos componentes es necesaria e inevitable.

Para finalizar, a modo de recomendación, es válida la propuesta de revisar las formas de candidaturas y de votación en el Ecuador, más aun cuando la votación por una sola lista ha sido significativamente alta a lo largo de los ańos: una posibilidad sería adoptar la propuesta original del plebiscito de 1997 y plantear un diseńo de listas cerradas y no bloqueadas. De igual forma, es necesario revisar las fórmulas electorales y ponderar una fórmula que se aplique para toda elección legislativa sin distinción alguna, considerando sobremanera, sus efectos proporcionales. Por último, con el afán de garantizar un real acceso de mujeres a cuerpos colegiados, entre las alternativas se podría plantear la generación de incentivos para que, en los procesos de democracia interna de las organizaciones políticas, el encabezamiento del total de listas a presentarse respete el principio de paridad. Del mismo modo, otra alternativa podría actuar al momento de la asignación de escańos; en este caso, el número de escańos ganadores podrían asignarse de forma paritaria, es decir, si una organización política gana dos escaños, el primer escaño iría a la candidata mujer más votada dentro de la lista y el segundo a su par masculino.

Es necesario revisar los efectos que actúan sobre los componentes de los sistemas electorales para comprender el camino que queda por 
recorrer en materia de inclusión, participación y representación. Es indiscutible que ningún sistema electoral es perfecto como también es cierto que todo sistema se encuentra en constante perfeccionamiento; lo importante, en este punto, es comprender que para diseñar caminos hay que conocer la estructuras de los obstáculos que se deben superar, así como las oportunidades con las que podemos contar y aprovechar para recorrerlos.

\section{Bibliografía}

Archenti, N. y María Inés Tula (2007). "Cuotas de género y tipo de lista en América Latina”. En Opinâo Pública Vol. 13 N.o 1: 185-218. Campinas, Junho.

Carey, J. y Matthew Shugart (1995). "Incentives to Cultivate a Personal Vote: a Rank Ordering of Electoral Formulas". En Electoral Studies Vol. 14 N.o 4: 417-439.

Cisneros, F. (2013). Cuaderno de Capacitación Electoral N.4: Elecciones Ecuador 2013: El sistema electoral para las elecciones a Presidentela y Vicepresidentela, Asamblea Nacional y Parlamento Andino. Quito: IDD.

Colomer, J. (2003). "Son los partidos los que eligen los sistemas electorales (o las leyes de Duverger cabeza abajo)". En Revista de Ciencia Politica N. 9: 39-63.

de Souza, C. (2013). "Paridad y cuotas electorales en Europa". En Pensar Vol. 18 N. ${ }^{\circ}$ : 72-97. Fortaleza.

Delegación Provincial Electoral de Pichincha (2013). Formulario para Análisis de Paquete Electoral. Quito.

Freidenberg, F. (2008). El sueño frustrado de la gobernabilidad: instituciones, actores y politica informal en Ecuador. Documentos CIDOB, América Latina N.o 24. Barcelona: CIDOB.

Instituto de la Democracia (2012). Cuaderno de Capacitación Electoral N. ${ }^{\circ}$ 1: Elecciones Ecuador 2013: Nuevas circunscripciones electorales y 
normativa para la inscripción y calificación de candidaturas. Quito: IDD.

IFE (2011). Elecciones presidenciales y legislativas en 18 paises de América Latina. México: IFE.

Lijphart, A. (1994). Electoral Systems and Party Systems. A Study of Twenty-Seven Democracies, 1945-1990. Oxford, versión española de 1995, Sistemas electorales y sistemas de partidos. Madrid: CEC.

Meier, P. (2008). "Bélgica: una buena práctica en perspectiva". En Sistemas electorales de cuotas de género y su aplicación en Europa. Drude Dahlerup y Lenita Freidenvall (eds.): 45-54. Bruselas: Parlamento Europeo.

Nohlen, D. (2007). "Sistemas electorales presidenciales y parlamentarios". En Tratado de derecho electoral comparado de América Latina. Dieter Nohlen, Daniel Zovatto, Jesús Orozco y José Thompson (comp.): 294-335. México: FCE, Instituto Interamericano de Derechos Humanos, Universidad de Heidelberg, International IDEA, Tribunal Electoral del Poder Judicial de la Federación, Instituto Federal Electoral. 2da. edición.

(2013). Gramática de los sistemas electorales: una introducción a la ingenieria de la representación. Quito: IDD.

Pachano, S. (1998). La representación caótica. Análisis del sistema electoral ecuatoriano. Quito: FLACSO.

(2007). "Partidos y sistemas de partidos en el Ecuador". En La politica por dentro: cambios y continuidades en las organizaciones politicas de los paises andinos: 161-211. Rafael Roncagliolo y Carlos Meléndez (eds.) Lima: IDEA.

Rae, D. W. (1971). The Political Consequences of Electoral Laws. New Haven \& Londres: Yale University Press.

Rule, W. (1987). "Electoral Systems, contextual factors and women's opportunity for election to partliament in twenty-three democracies”. En Western Political Quarterly Vol. 40 N. ${ }^{3}$, Sep.

Sartori, G. (1986). "The Influence of Electoral Systems. Faulty Laws or Faulty Methods" En Electoral Laws and Their Political Consequences. 
Bernard Grofman y Arend Lijphart (eds.) New York: Agathon Press Mimeo.

(2012) Elementos de teoría politica. Madrid: Alianza Editorial. Taagapera, R. y Matthew S. Shugart (1989). Seats and Votes: The Effects of Determinants of Electoral Systems. New Haven: Yale University Press.

Normativa y documentos oficiales

Constitución de la República del Ecuador (2008). Asamblea Constituyente. Ciudad Alfaro.

Consejo Nacional Electoral (2013). Resultados Electorales. Quito.

Ley Orgánica Electoral y de Organizaciones Politicas de la República del Ecuador, Código de la Democracia (2009). Asamblea Nacional. Quito: Suplemento R.O. 578 del 27 abril.

Ley Orgánica de Elecciones (2000). R.O. 117 del 11 julio. 